\title{
MIMO Frequency Sampling Filters for Modelling of MIMO System Applications"
}

\author{
Muhammad Hilmi R.A. Aziz ${ }^{1}$ \& Rosmiwati Mohd-Mokhtar ${ }^{2}$ \\ ${ }^{1}$ School of Electrical \& Electronic Engineering, Universiti Sains Malaysia, \\ Engineering Campus, 14300, Nibong Tebal, Pulau Pinang, Malaysia \\ ${ }^{2}$ School of Electrical \& Electronic Engineering, Universiti Sains Malaysia, \\ Engineering Campus, 14300, Nibong Tebal, Pulau Pinang, Malaysia \\ Email: rosmiwati@ieee.org
}

\begin{abstract}
In the modelling of a system based on a system identification approach, data acquisition is the first procedure that must be carried out. The data acquisition process from a real system typically yields large amounts of data. This may lead to unacceptable computational time during the identification process. Raw data may also suffer severe noise disturbance, especially in the high frequency region. In addition, bias estimation will occur if one only considers direct identification from a closed-loop system. To overcome this problem, in this paper a multivariable frequency sampling filter approach is introduced. Multi-input-multi-output (MIMO) raw data are analyzed in order to obtain only relevant and meaningful parameters that describe the empirical model of the analyzed data. By performing this procedure, compressed, cleaned and unbiased data are produced. The efficacy of the MIMO frequency sampling filters was demonstrated by compressing two sets of data: $\mathrm{pH}$ neutralization process data and steam generator plant data. The results show that the amount of raw data was successfully compressed and that the output was ready for identification purposes with less computational time, i.e. they could be further used to develop a model of the system, to conduct time and frequency response analysis, and also for developing a new control system design.
\end{abstract}

Keywords: Data compression; frequency sampling filters; multivariable process; nonparametric model; system identification.

\section{$1 \quad$ Introduction}

System identification is based on study and analysis of input and output data collected from a system. To perform this procedure, a data acquisition process is unavoidable. Data acquisition from a real system typically yields large amounts of discrete-time data. This may lead to unacceptable computational time during the identification process. Also, the raw data may contain complex system

\footnotetext{
* This paper is an extended version of the paper published by the authors in Int. Conf. on Electrical Engineering \& Informatics (ICEEI 2011).
}

Received November $3^{\text {rd }}, 2013$, Revised January $14^{\text {th }}, 2013$, Accepted for publication January $21^{\text {st }}, 2013$. Copyright @ 2013 Published by ITB Journal Publisher, ISSN: 2337-5779, DOI: 10.5614/j.eng.technol.sci.2013.45.1.6 
disturbance information, which may require a sophisticated optimization algorithm in order to achieve the desired results [1-5]. In addition, for data collected from a closed-loop system, bias estimation will occur if one only considers direct identification. An open-loop direct identification approach is applicable only if the appearance of noise disturbance is not significant and can be neglected. Fortunately, this problem can be solved by introducing a frequency sampling filter (FSF) within the first stage of the identification process [4-8].

FSF is a discrete-time model. The FSF approach is useful in addressing the problem of large amounts of data collected from a system. By performing an FSF procedure, compressed, cleaned and unbiased data can be obtained from the system. The information gathered from this step can be further used to develop a model of the system. Besides that, analysis and observation can be done for optimization and controller design purposes. The smaller amount of computational time and load due to the smaller number of data being processed will also help in improving the overall performance of the required procedures. The plot for step response and frequency response data obtained after performing frequency sampling can also be used to analyze the performance of the observed system [4-8].

The FSF structure was first introduced into the field of system identification and automatic control by Bitmead and Anderson [9], Parker and Bitmead [10]. Research in the signal-processing field is looking at designing modified frequency-sampling filters that account for finite word-length effects [11]. In reference [12], the authors have combined this model structure with a standard least-squares estimator in order to directly obtain an estimate of the process frequency response with a frequency-domain statistical bound on the model uncertainty. FSF models have been used to estimate step response models along with their corresponding time domain confidence bounds [13].

The step response (invariant in both discrete and continuous time) obtained from FSF models is gaining much interest for use in system identification, since it provides information regarding the stability of the system and the ability to reach stationary state. It also has a transparent representation in terms of gain, time delay and time constant. It is widely viewed as a precursor to the design of further experiments, as an indicator for the collection of more input-output data, and as a subsequent procedure for regression-based techniques to obtain more accurate models $[6,14]$.

The use of FSF as data compression method can be found in various single input single output (SISO) applications [7, 8, 15-17]. However, most systems in control engineering are multivariable in nature. The control theory for 
multivariable systems certainly finds immediate application in a wide variety of problems. The control of multivariable processes is complicated due to the coupling that exists between the control inputs and the outputs. For systems that have more than one input and output variable (especially for ill-conditioned processes), those inputs/outputs must be analysed together in order to foresee the correlation between them.

Thus, the aim of this paper is to develop a new multi-input multi-output FSF algorithm. This novel contribution will facilitate MIMO system identification, in terms of providing cleaned and unbiased MIMO compressed data. In addition, the analysis of a MIMO system via the MIMO approach will definitely give clearer observations since the involvement of all the input/output variables can be examined inclusively.

In brief, this paper is organized as follows. Section 2 describes the frequency sampling filter model. Details on how to obtain step and frequency response data via the FSF model approach will be elaborated in Section 3. Then, the performance and results of the proposed approach will be elaborated in section 4. Here, two system examples will be used: a $\mathrm{pH}$ neutralization process and a steam generator process. Finally, Section 5 concludes the paper.

\section{$2 \quad$ Frequency Sampling Filter Model}

The FSF approach used in this paper is originally obtained from [14,18]. The FSF model can be identified as

$$
y(k)=G(z) u(k)+v(k)
$$

where $u(k), y(k)$ are input and output signal respectively. $v(k)$ is the zero mean disturbance term. $G(z)$ is obtained from linear transformation of the FIR model. With the assumptions that the process being identified is stable, linear and time invariant, the FIR transfer function model is

$$
G(z)=\sum_{i=0}^{n-1} h_{i} z^{-i}
$$

To derive the FSF model, we make use of the inverse Discrete Fourier Transform (DFT) relationship between the process frequency response and its impulse response, under the assumption that $n$ is an odd number

$$
h_{i}=\frac{1}{n} \sum_{m=-\frac{n-1}{2}}^{\frac{n-1}{2}} G\left(e^{j \frac{2 \pi n}{n}}\right) e^{j \frac{2 \pi n i}{n}}
$$


This relationship maps a set of discrete-time frequency response coefficients, $G\left(e^{j \frac{2 \pi n}{n}}\right), m=0, \pm 1, \pm 2, \ldots, \pm \frac{n-1}{2}$ into the set of discrete-time unit impulse response coefficients, $h_{i}, i=0, \ldots, n-1$. Substituting (3) into (2) gives

$$
G(z)=\sum_{i=0}^{n-1} \frac{1}{n} \sum_{m=-\frac{n-1}{2}}^{\frac{n-1}{2}} G\left(e^{j \frac{2 \pi n}{n}}\right) e^{j \frac{2 \pi n i}{n}} z^{-i}
$$

Interchanging the summations in (4) gives the transfer function in its FSF model form

$$
G(z)=\sum_{m=-\frac{n-1}{2}}^{\frac{n-1}{2}} G\left(e^{j \frac{2 \pi n}{n}}\right) \frac{1}{n} \frac{1-z^{-n}}{1-e^{j \frac{2 \pi n}{n}} z^{-1}}
$$

where

$$
\sum_{i=0}^{n-1} e^{j \frac{2 \pi n i}{n}} z^{-i}=\frac{1-z^{-n}}{1-e^{j \frac{2 \pi n}{n}} z^{-1}}
$$

Define a set of transfer functions extracting from (5)

$$
H^{m}(z)=\frac{1}{n} \frac{1-z^{-n}}{1-e^{j \frac{2 \pi m}{n}} z^{-1}}
$$

for $m=0, \pm 1, \pm 2, \ldots, \pm \frac{n-1}{2}$, the above equation is referred to as the $m$-th FSF with the centre frequency of the $m$-th filter at $\frac{2 \pi m}{n}$ radians. Let $z=e^{j \omega}$, (5) will become

$$
G\left(e^{j \omega}\right)=\sum_{m=-\frac{n-1}{2}}^{\frac{n-1}{2}} G\left(e^{j \frac{2 \pi n}{n}}\right) \frac{1}{n} \frac{1-e^{-j \omega n}}{1-e^{j \frac{2 \pi n}{n}} e^{-j \omega}}
$$

At $\omega=\omega_{m}$, the following condition holds

$$
\begin{aligned}
& H^{a}\left(e^{j \omega}\right)=0 \quad \text { for } \quad a \neq m \\
& H^{a}\left(e^{j \omega}\right)=1 \quad \text { for } \quad a=m
\end{aligned}
$$


where $a$ is an integer like $m$ in the range $\left[-\frac{n-1}{2}, \frac{n-1}{2}\right]$. In this case, the value of the process frequency response in (8) is reduced to the value of the process frequency response coefficient $G\left(e^{j \frac{2 \pi n}{n}}\right)$.

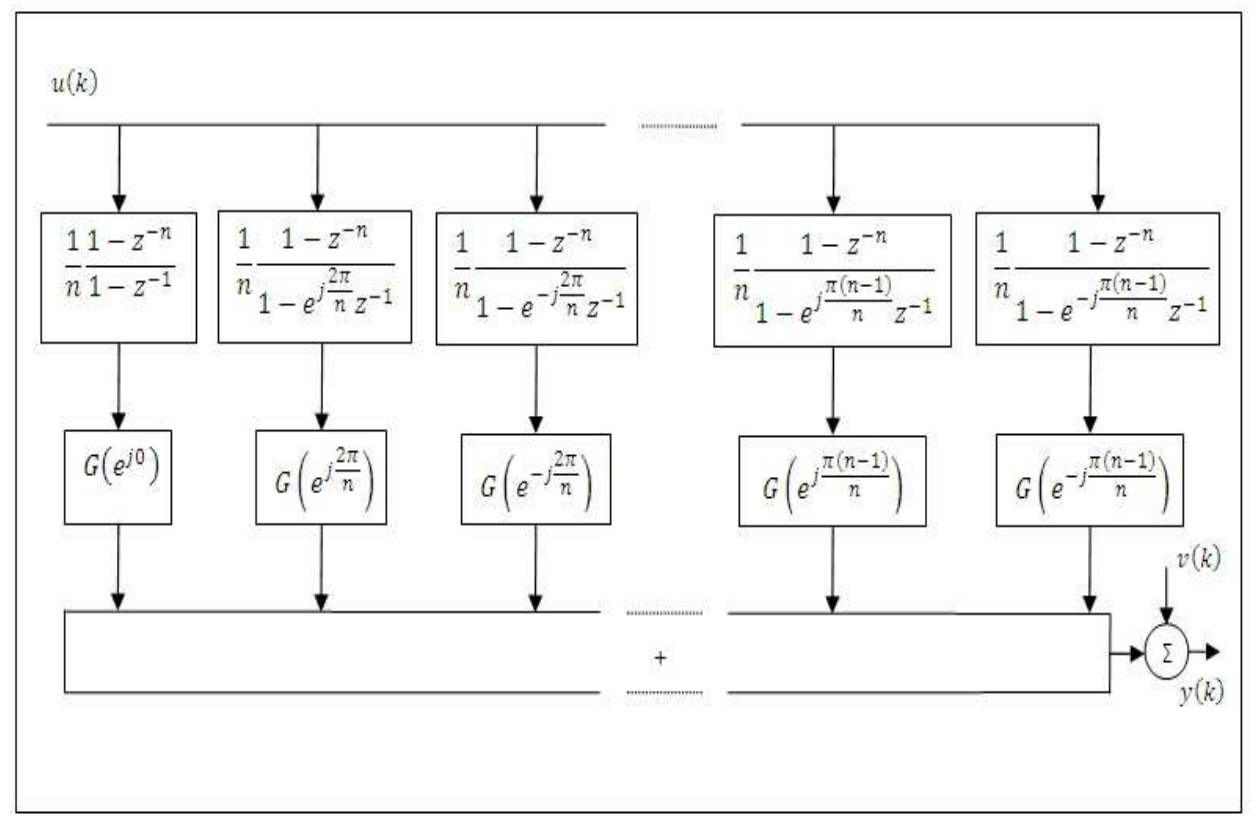

Figure 1 FSF model structure (adapted from [14]).

Given the discrete input signal, $u(k)$, the discrete measured output signal, $y(k)$, and the disturbance, $v(k)$, the FSF model can be explained in a block diagram, as shown in Figure 1. Figure 1 shows a block diagram of the frequency sampling filter model being used to represent the process. The process input first passes through the set of frequency sampling filters arranged in parallel. Then, the output of each filter is weighted by the discrete-time process frequency response evaluated at the corresponding centre frequency. Finally, the weighted filter outputs are summed to form the noise-free process output. The FSF filters are narrow band limited around their respective centre frequencies. All the filters have identical frequency responses except for the location of their centre frequencies.

Define the parameter vector as 


$$
\theta=\left[\begin{array}{c}
G(0) \\
G\left(e^{j \frac{2 \pi}{n}}\right) \\
G\left(e^{-j \frac{2 \pi}{n}}\right) \\
\vdots \\
G\left(e^{j\left(\frac{(n-1) \pi}{n}\right)}\right) \\
G\left(e^{-j\left(\frac{(n-1) \pi}{n}\right)}\right)
\end{array}\right]
$$

and its corresponding vector regressor vector as

$$
\phi(k)=\left[\begin{array}{c}
f_{0}(k) * u(k) \\
f_{1}(k) * u(k) \\
f_{-1}(k) * u(k) \\
\vdots \\
f_{\frac{n-1}{2}}(k) * u(k) \\
f_{-\frac{n-1}{2}}(k) * u(k)
\end{array}\right]
$$

where $\left({ }^{\mathrm{T}}\right)$ denotes the transpose matrix and $f_{m}(k)$ is defined according to (7) as

$$
f_{m}(k)=H^{m}(z)
$$

Thus, (1) can be rewritten as

$$
y(k)=\theta^{\mathrm{T}} \phi(k)+v(k)
$$

For $N$ data measurements, (12) can also be written in matrix form as

$$
Y=\Theta \Phi+V
$$

Eq. (13) can then be used to solve the least squares estimate of $\Theta$ given by

$$
\hat{\Theta}=\left(\Phi_{N}^{\mathrm{T}} \Phi_{N}\right)^{-1} \Phi_{N}^{\mathrm{T}} Y_{N}
$$

which minimizes the performance index of the form

$$
J(N, \hat{\Theta})=\sum_{k=0}^{N}|Y-\Pi \Phi|^{2}
$$


The matrix $\left(\Phi_{N}^{\mathrm{T}} \Phi_{N}\right)$ is called the correlation matrix and the invertibility condition on this matrix is sometimes called the sufficient excitation condition for parameter estimation. In order to obtain a proper FSF parameter optimization, the least squares model estimates based on PRESS (predicted residual sum of squares) computation is used. The PRESS criterion will ensure that the FSF model has the greatest predictive capability among all candidate models [19].

\subsection{Multivariable FSF}

In order to take full advantage of the orthogonal decomposition algorithm for parameter estimation, we chose the multivariable system to be identified. For the MIMO system, $p$ inputs are denoted as $u_{1}(k), u_{2}(k), \ldots, u_{p}(k)$ and $q$ outputs are denoted as $y_{1}(k), y_{2}(k), \ldots, y_{q}(k)$, the times to steady state for each subsystem are given by $\mathrm{N}_{1}, \mathrm{~N}_{2}, \ldots, \mathrm{N}_{p}$, and the reduced orders for each subsystem are represented by its own FSF model are chosen to be $n_{1}, n_{2}, \ldots, n_{p}$. For the MISO system, the $q$ outputs are denoted as $y(k)$. For the multivariable system, the matrix representation is dictated as follows:

1. The first input $u_{1}(k)$ is passed through a set on $n_{1}$ FSF based on $\mathrm{N}_{1}$, to form the first $n_{1}$ columns of the data matrix.

2. The next input is passed accordingly to form the next columns of the data matrix.

3. The associated parameters comprising of all the subsystems will be justified in the multivariable form.

4. The least squares algorithm is then applied to estimate the FSF model parameters with respect to each subsystem.

\subsection{The PRESS Criterion}

The idea of PRESS is to set aside each data point, estimate a model using the rest of the data, and then evaluate the prediction error at the point that was removed. The PRESS statistic can be applied as a criterion for model structure detection in dynamic system identification.

Define the prediction error as

$$
\begin{aligned}
e_{-k}(k) & =y(k)-\hat{\theta}^{\mathrm{T}} \phi(k) \\
& =y(k)-\hat{y}_{-k}(k)
\end{aligned}
$$


where $e_{-k}(k), k=1,2, \ldots, N$ are called the PRESS residuals and $\hat{\theta}$ has been estimated according to (14) without including $\phi(k)$ and $y(k)$. The PRESS residuals $e_{-k}(k)$ represent the true prediction errors and can be calculated according to the following equation

$$
e_{-k}(k)=\frac{e(k)}{1-\phi(k)^{\mathrm{T}}\left(\Phi^{\mathrm{T}} \Phi\right)^{-1} \phi(k)}
$$

The PRESS statistic is defined as

$$
\text { PRESS }=\sum_{k=1}^{N} e_{-k}(k)^{2}
$$

The average PRESS is calculated as

$$
\text { PRESS }_{a v}=\sqrt{\frac{\sum_{k=1}^{N} e_{-k}(k)^{2}}{N-1}}
$$

Both (18) and (19) provide measures of the predictive capability of the estimated model. In terms of model structure selection, the chosen structures are based on the smallest PRESS value.

\section{Step and Frequency Response Estimate Using FSF Model}

In the estimation of step response, the description of the system using the FSF model can be described as follows:

$$
y(k)=\sum_{m=-\frac{n-1}{2}}^{\frac{n-1}{2}} G\left(e^{j \frac{2 \pi n}{n}}\right) H^{m}(z) u(k)+v(k)
$$

where for a suitable choice of $G\left(e^{j \frac{2 \pi n}{n}}\right)$ and $H^{m}(z)$ are defined as in (4) and (7) respectively. Upon obtaining the estimate of the frequency response parameters (according to the FSF model and PRESS criterion), the estimate of the step response at sampling instant $m$ can be expressed by

$$
\hat{g}_{m}=\sum_{i=0}^{m-1} \hat{h}_{i}
$$

where the estimated impulse response coefficients $\hat{h}_{0}, \hat{h}_{1}, \hat{h}_{2}, \ldots, \hat{h}_{m-1}$ are related to frequency response via 


$$
\left.\hat{h}_{i}=\frac{1}{N} \sum_{m=-\frac{n-1}{2}}^{\frac{n-1}{2}} \hat{G} e^{j \frac{2 \pi n n}{n}}\right) e^{j \frac{2 \pi n i}{n}}
$$

By substituting (22) into (21), the estimated step response coefficient can be rewritten as

$$
\hat{g}_{m}=\sum_{m=-\frac{n-1}{2}}^{\frac{n-1}{2}} \hat{G}\left(e^{j \frac{2 \pi n}{n}}\right) \frac{1}{N} \frac{1-e^{j \frac{2 \pi n}{n}(m+1)}}{1-e^{j \frac{2 \pi n}{n}}}
$$

Although the FSF approach is cast in the discrete-time domain and the corresponding $z$-transform domain, the resultant model can be used to obtain continuous time step response. The system impulse response $\hat{g}(t)$ can be approximately computed using the continuous time equivalent as

$$
\hat{g}(t) \approx \hat{g}_{f s f}(t)=\sum_{m=-\frac{n-1}{2}}^{\frac{n-1}{2}} \hat{\theta}_{m} \hat{h}_{m}(t)
$$

and

$$
\hat{h}_{m}(t)=\frac{1}{T} e^{j \frac{2 \pi m i}{T}} \quad \text { for } \quad t<T \Delta
$$

where $T$ is a sampling period. The step response is determined as

$$
y_{s}(t)=\int_{0}^{t} \hat{g}(\tau) d \tau
$$

The frequency response estimate is obtained over a transformation of $\bar{z}=e^{j \omega \Delta}$. Thus, in frequency domain form, the equations can be rewritten as

$$
\begin{aligned}
& G(j \omega) \approx G_{f s f}(j \omega)=\sum_{k=-\frac{n-1}{2}}^{\frac{n-1}{2}} \theta_{k} H_{k}(j \omega) \\
& H_{k}(j \omega)=\bar{H}_{k}\left(e^{j \omega \Delta}\right) \text { for } \omega<\aleph \Omega
\end{aligned}
$$

where $n$ is odd and the frequency sampling interval $\Omega=\frac{2 \pi}{T}, H_{k}(z)$ is the $k$-th FSF and $\theta_{k}$ is the corresponding (complex) parameter for the frequency range of $0 \leq \omega \leq \aleph \Omega$. 


\subsection{Confidence Bounds for the Step Response Model}

The confidence bounds for the step response model derived from the FSF model using (23) can be obtained. The basic idea is to represent the step response coefficients as a linear transformation of the estimated FSF parameters.

Theorem 1. Let the estimated step response be represented by:

$$
\hat{g}_{m}=S(l) \hat{\theta}
$$

where $S(l)=\left[\begin{array}{llll}S(0, l) & S(1, l) & \cdots & S\left(-\frac{n-1}{2}, l\right)\end{array}\right]$ with $S(l, m)$ defined as

$$
S(m, l)=\frac{1}{N} \frac{1-e^{j \frac{2 \pi n}{n}}(l+1)}{1-e^{j \frac{2 \pi n}{n}}}
$$

By considering the following assumptions:

A1. The process is stable, linear and time invariant with finite settling time $T_{s}$ and the parameter $n$ is chosen to be greater than or equal to $\frac{T_{s}}{\Delta t}$.

A2. The disturbance $v$ is zero mean, normally distributed white noise with variance $\sigma^{2}$.

A3. $N=n$, or $N$ is chosen such that the neglected frequency parameters are negligible in magnitude relative to the parameters being retained in the model.

The error between the true process step response weight $g_{m}$ and the estimated step response weight $\hat{g}_{m}$ is bounded by

$$
\left|\hat{g}_{m}-g_{m}\right| \leq p * \delta(l)
$$

with probability $P(p)$, where $\delta(l)$ is given by

$$
\delta(l)^{2}=S(l)\left(\Phi^{\mathrm{T}} \Phi\right)^{-1} S^{\mathrm{T}}(m) \sigma^{2}
$$

Based on assumptions A1-A3, the following properties of the least square estimate $\hat{\theta}$ obtained from (13) can be stated:

- Bias: The estimate $\hat{\theta}$ is unbiased.

- Variance: The covariance of the parameter estimates is given by

$$
E\left[(\hat{\theta}-\theta)(\hat{\theta}-\theta)^{\mathrm{T}}\right]=\left(\Phi^{\mathrm{T}} \Phi\right)^{-1} \sigma^{2}
$$


- Distribution properties: Since only a linear operation is involved in estimating the parameters, $\hat{\theta}$ will follow a normal distribution.

Proof 1. Under assumption A1, the true process step response can be represented by

$$
g_{m}=S(l) \theta
$$

From assumptions A1-A3, we know that $\hat{\theta}$ is an unbiased and normally distributed estimate of $\theta$. Therefore, $\hat{g}_{m}$ is an unbiased and normally distributed estimate of $g_{m}$. The variance of the estimated step response coefficient at the sampling instant $m$ is given by

$$
\begin{aligned}
E\left[\left(\hat{g}_{m}-g_{m}\right)^{2}\right] & =E\left[S(l)(\hat{\theta}-\theta)(\hat{\theta}-\theta)^{\mathrm{T}} S^{\mathrm{T}}(l)\right] \\
& =S(l)\left(\Phi^{\mathrm{T}} \Phi\right)^{-1} S^{\mathrm{T}}(l) \sigma^{2} \\
& =\delta(l)^{2}
\end{aligned}
$$

and the bounds in (31) follow directly.

Applying (31), the trajectory of the true step response $g_{m}$ for $m=0,1, \ldots, n-1$ lies inside the envelope given by $\hat{g}_{m} \pm p * \delta(l)$ with probability $P(p)$. This envelope provides the confidence bound on the estimated step response model.

\section{$4 \quad$ Results and Discussion}

The overall flow of the process is shown in Figure 2. Two sets of multivariable real system data have been used to demonstrate the efficacy of the developed multivariable FSF. Firstly, multi input single output (MISO) data from a simulation of a $\mathrm{pH}$ neutralization process in a stirring tank. Secondly, multi input multi output (MIMO) data from a model of a steam generator at a power plant. Both of these data sets are industrial system process data obtained from [20]. 


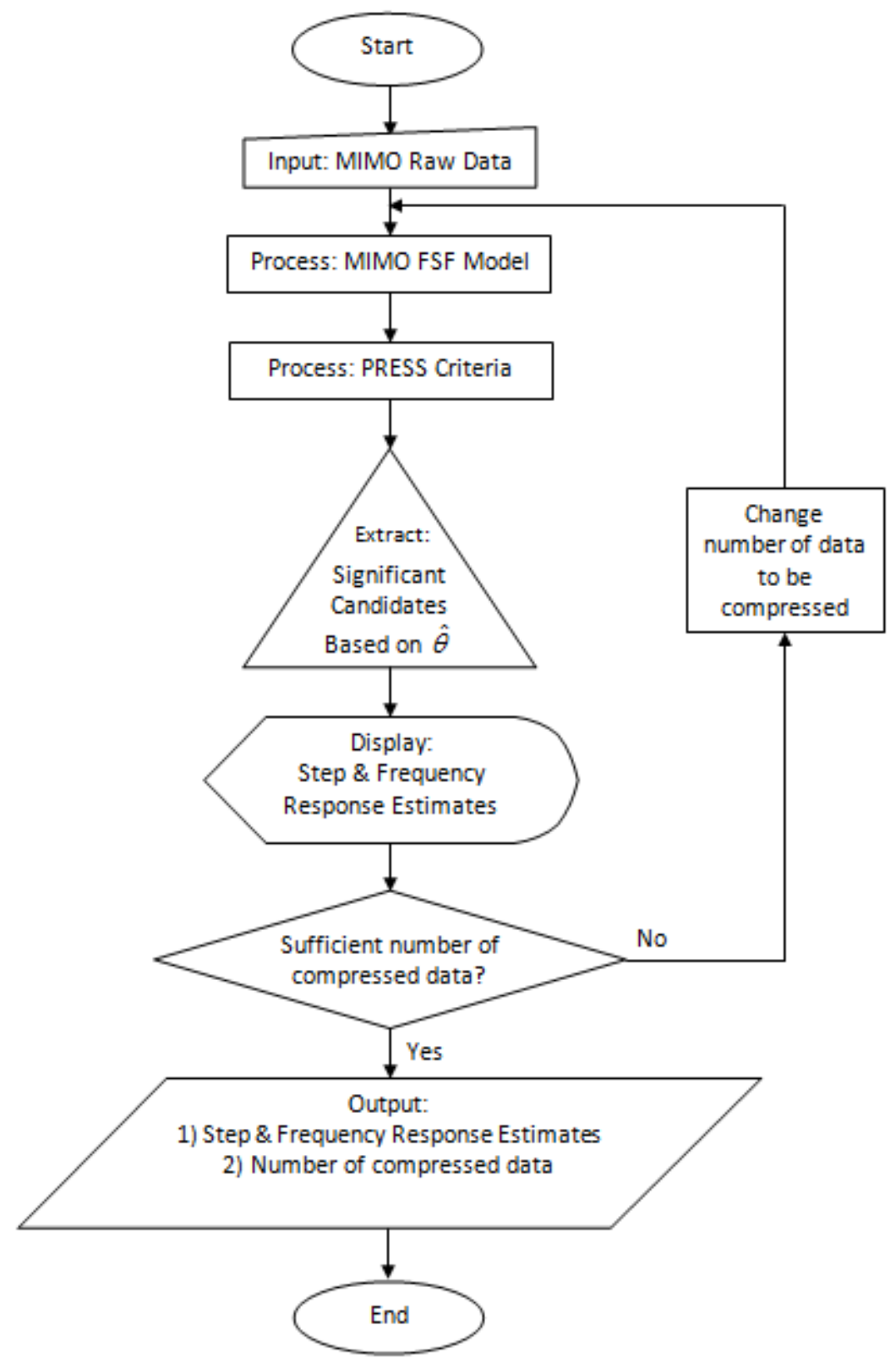

Figure 2 Overall procedure. 


\subsection{Example 1 - pH Neutralization Data}

The volume of the tank used in the $\mathrm{pH}$ neutralization process was 1100 litres. This process is a highly non-linear system. The acid and the base solution were mixed together in a constant-volume stirring tank and the $\mathrm{pH}$ neutralization process data were simulated. The concentration of the acid solution (HAC) was $0.0032 \mathrm{Mol} / \mathrm{l}$ and the concentration of the base solution $(\mathrm{NaOH})$ was $0.05 \mathrm{Mol} / \mathrm{l}$. About $N=2000$ data were measured in $10 \mathrm{~s}$. The inputs were acid solution flow in litres and base solution flow in litres, while the output of the system was the $\mathrm{pH}$ solution in the tank (See Figure 3).
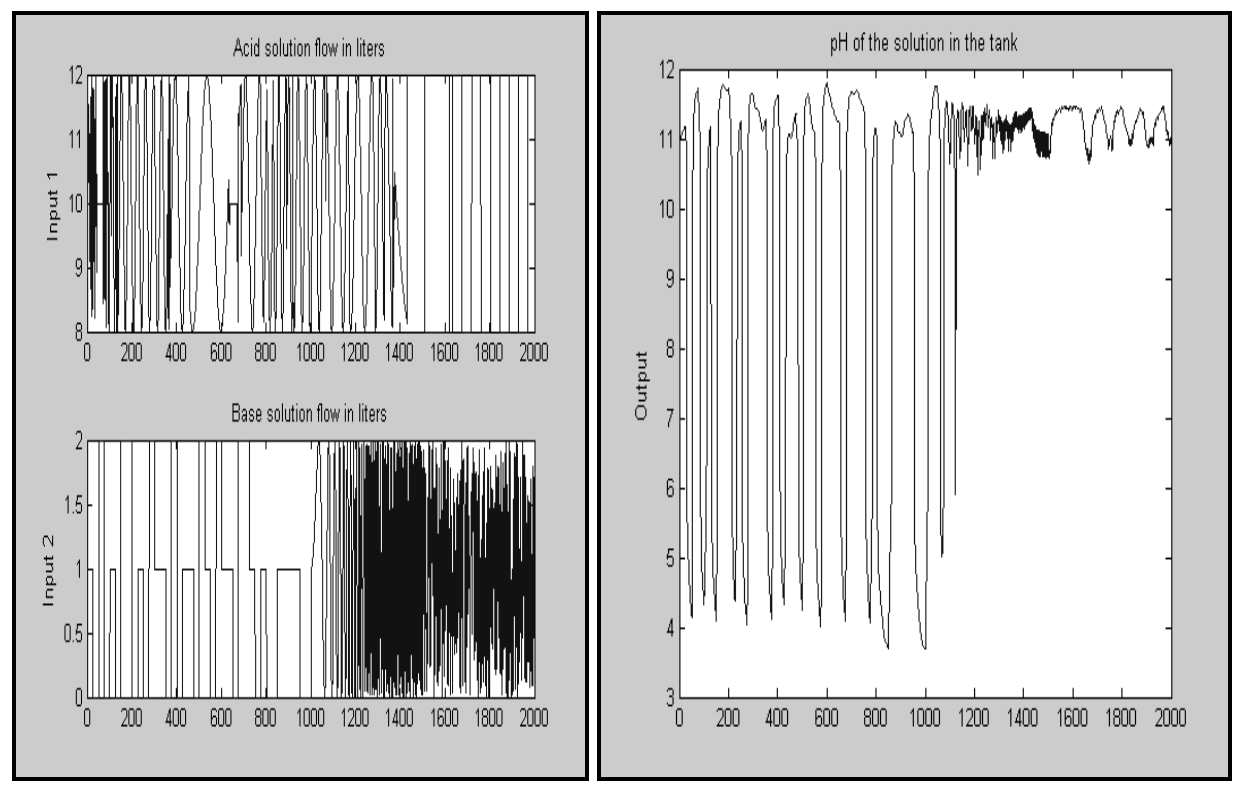

Figure 3 Input and output plot for $\mathrm{pH}$ neutralization data system.

The two inputs and one output of the $\mathrm{pH}$ neutralization system were defined by the following configuration

$$
[y(t)]=\left[\begin{array}{ll}
G_{11} & G_{12}
\end{array}\right]\left[\begin{array}{l}
u_{1}(t) \\
u_{2}(t)
\end{array}\right]
$$

Figure 4 and Figure 5 illustrate the step response and the frequency response obtained from the process data. The number of successfully compressed data of the process is described in Table 1. 


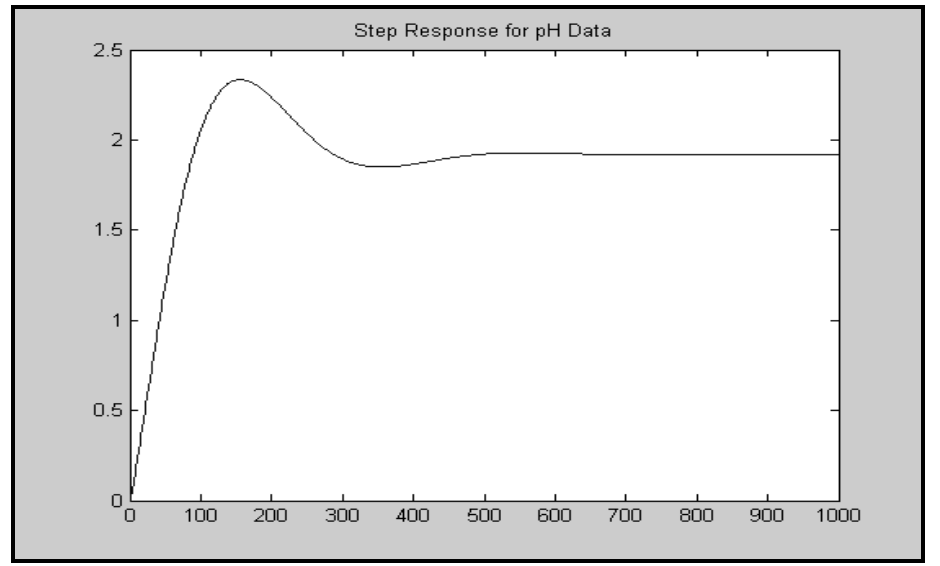

Figure 4 Step response obtained from FSF model of Example 1.

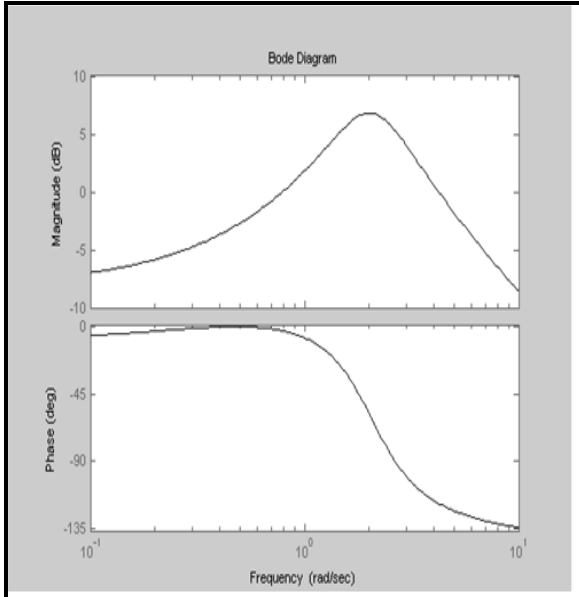

(a)

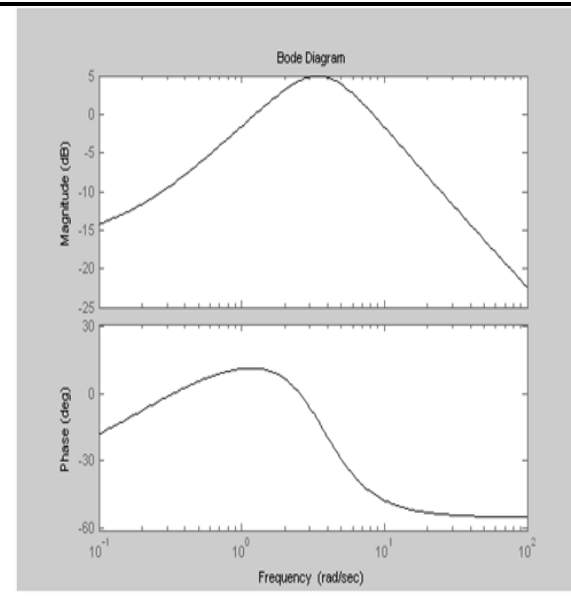

(b)

Figure 5 Frequency response obtained from FSF model of Example 1; (a) $\mathrm{G}_{11}$ (b) $\mathrm{G}_{12}$

\subsection{Example 2 - Steam Generator Data}

These data come from a model of a steam generator at the Abbott Power Plant in Champaign IL (refer to Figure 6). A detailed description of this model can be obtained from [21]. About $N=9600$ data were measured in $3 s$. The inputs for this model were fuel (scaled 0-1), air (scaled 0-1), reference level in inches, and disturbance defined by load level. The outputs were drum pressure, excess oxygen in exhaust gases (\%), level of water in the drum and steam flow $(\mathrm{Kg} / \mathrm{s})$. Figure 7 illustrates the inputs and the outputs of this plant. The four inputs and 
four outputs of the steam generator system were defined by the following configuration

$$
\left|\begin{array}{l}
y_{1}(t) \\
y_{2}(t) \\
y_{3}(t) \\
y_{4}(t)
\end{array}\right|=\left[\begin{array}{llll}
G_{11} & G_{12} & G_{13} & G_{14} \\
G_{21} & G_{22} & G_{23} & G_{24} \\
G_{31} & G_{32} & G_{33} & G_{34} \\
G_{41} & G_{42} & G_{43} & G_{44}
\end{array}\right]\left[\begin{array}{l}
u_{1}(t) \\
u_{2}(t) \\
u_{3}(t) \\
u_{4}(t)
\end{array}\right]
$$

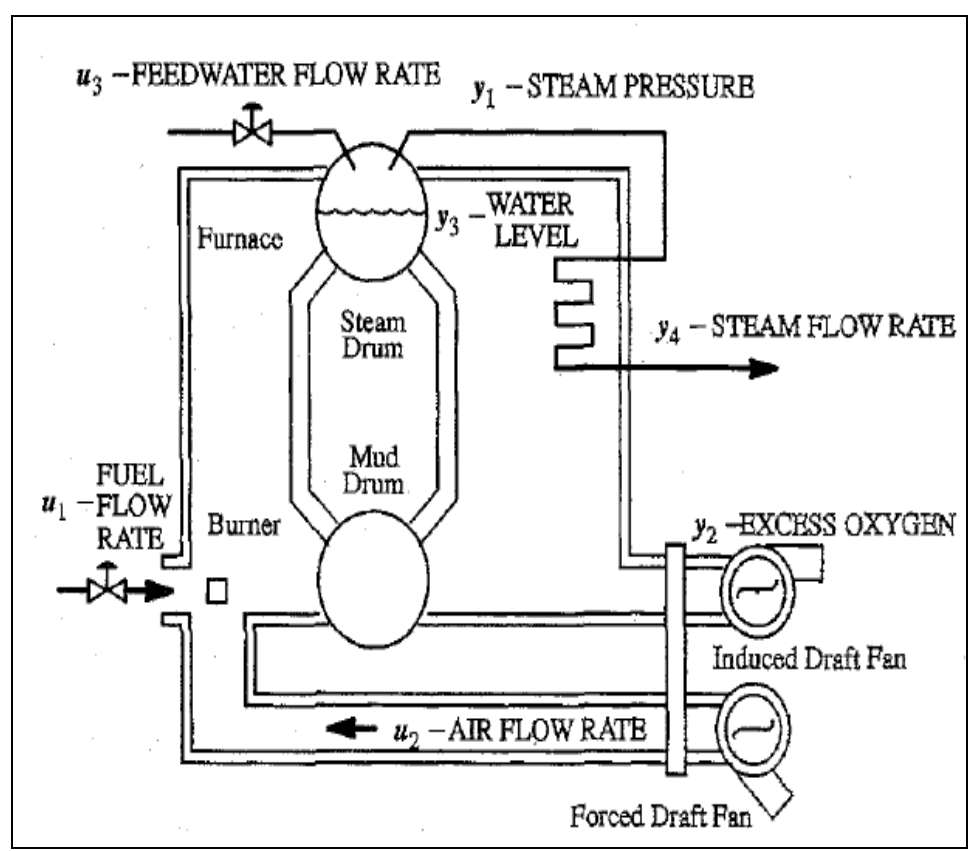

Figure 6 Industrial steam generator plant (adapted from [12]).

Step response and frequency response obtained for these inputs and outputs data are shown in Figure 8 and Figure 9. The number of successfully compressed data is shown in Table 1 .

Table 1 Number of data before and after performing FSF.

\begin{tabular}{ccc}
\hline Types of data & $\begin{array}{c}\text { Number of raw } \\
\text { data }\end{array}$ & $\begin{array}{c}\text { Number of } \\
\text { compressed data }\end{array}$ \\
\hline MISO - Example 1 & 2000 & 1000 \\
MIMO - Example 2 & 9600 & 3000 \\
\hline
\end{tabular}




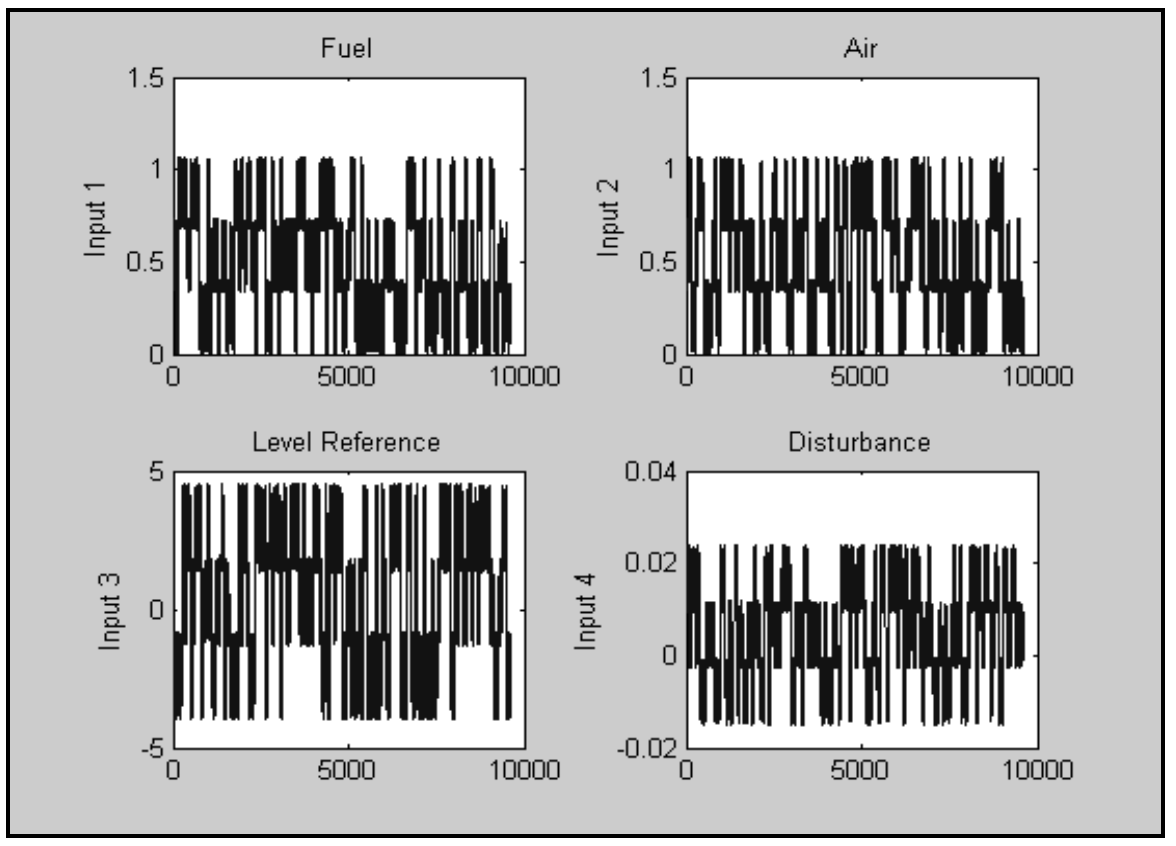

(a)

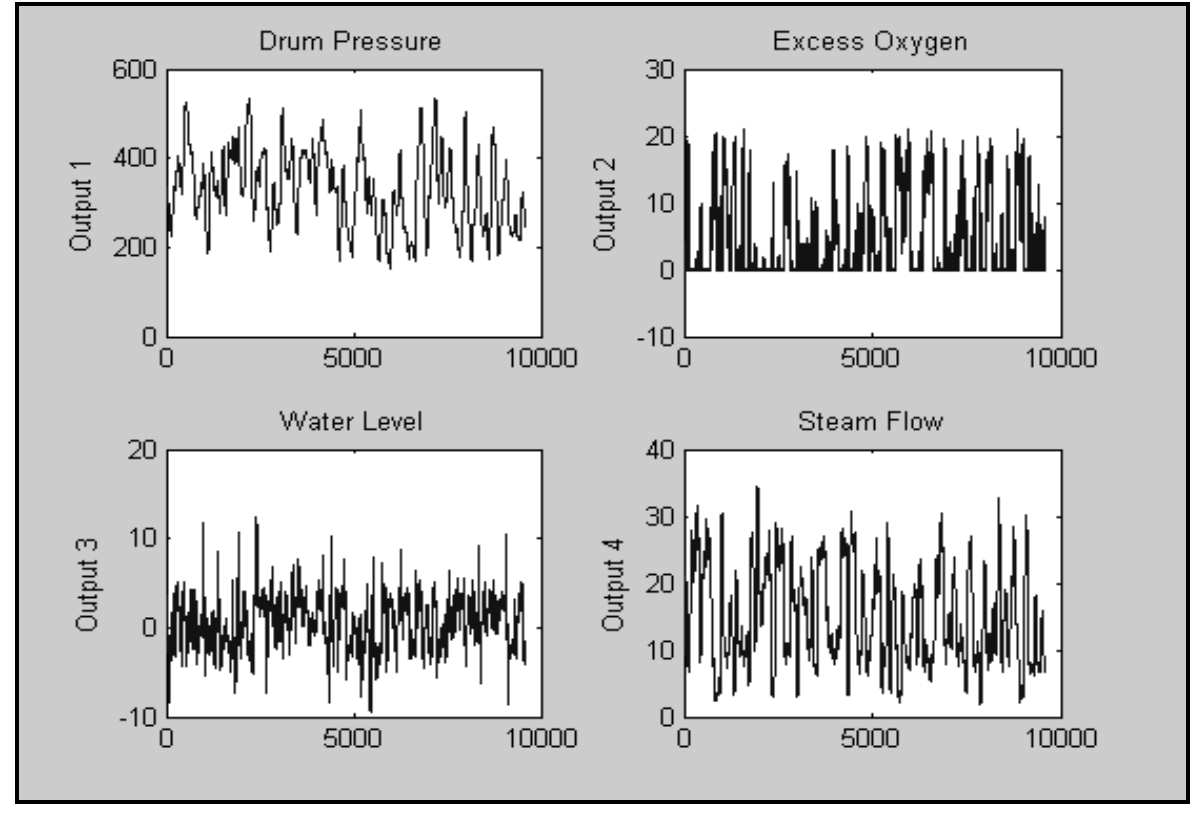

(b)

Figure 7 Input (a) and Output (b) plot for steam generator data system. 


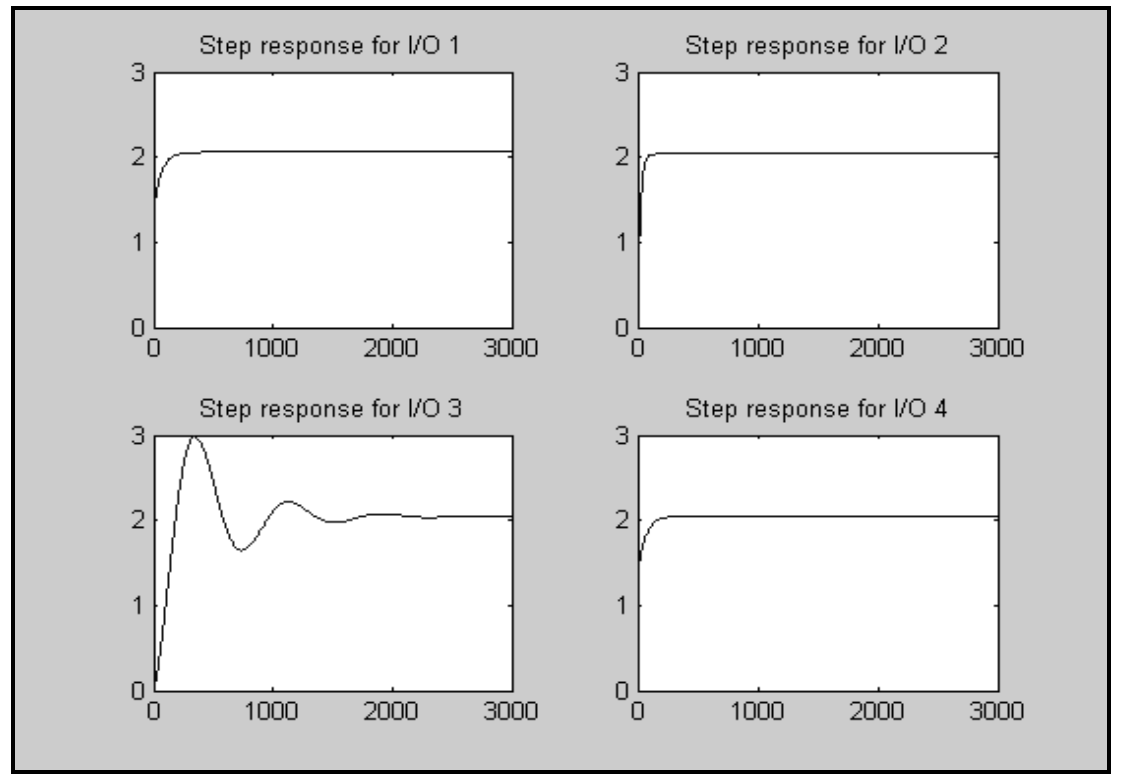

Figure 8 Step response obtained from FSF model of Example 2.

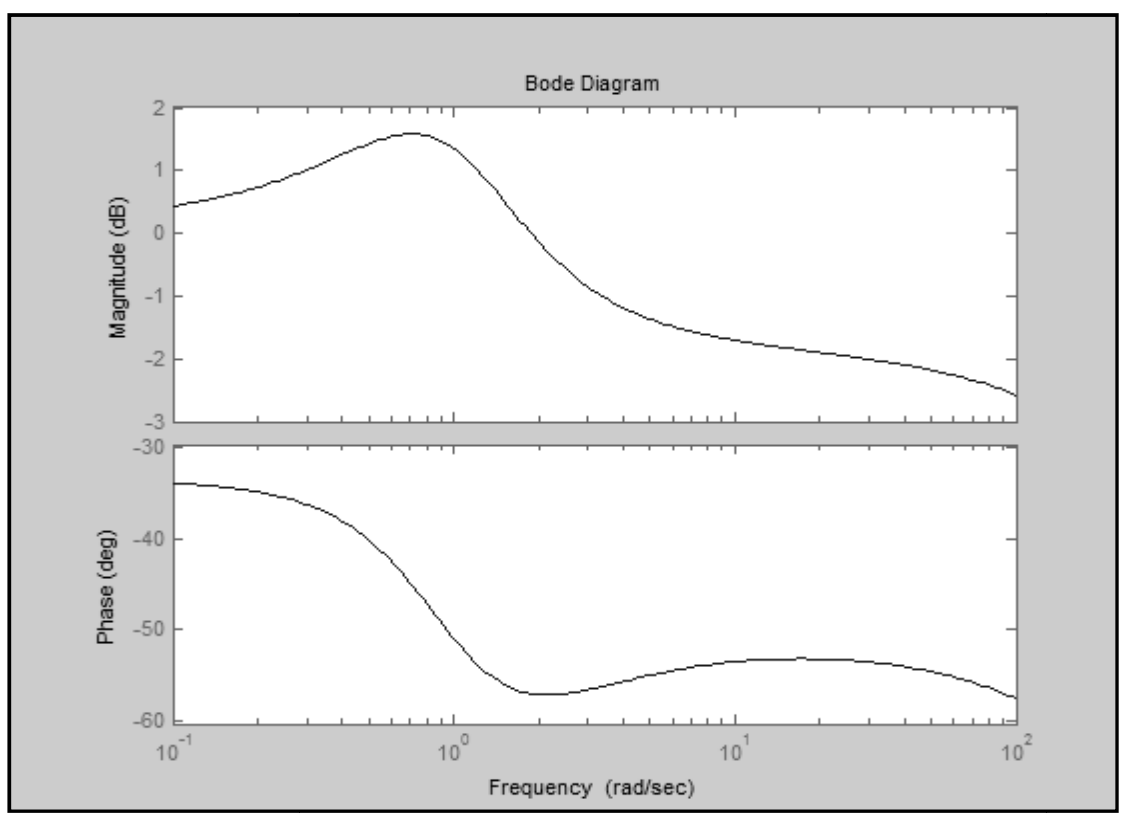

(a)

Figure 9 Frequency response obtained from FSF model of Example 2 for (a) Input/Output 1 (b) Input/Output 2 (c) Input/Output 3 and (d) Input/Output 4. 


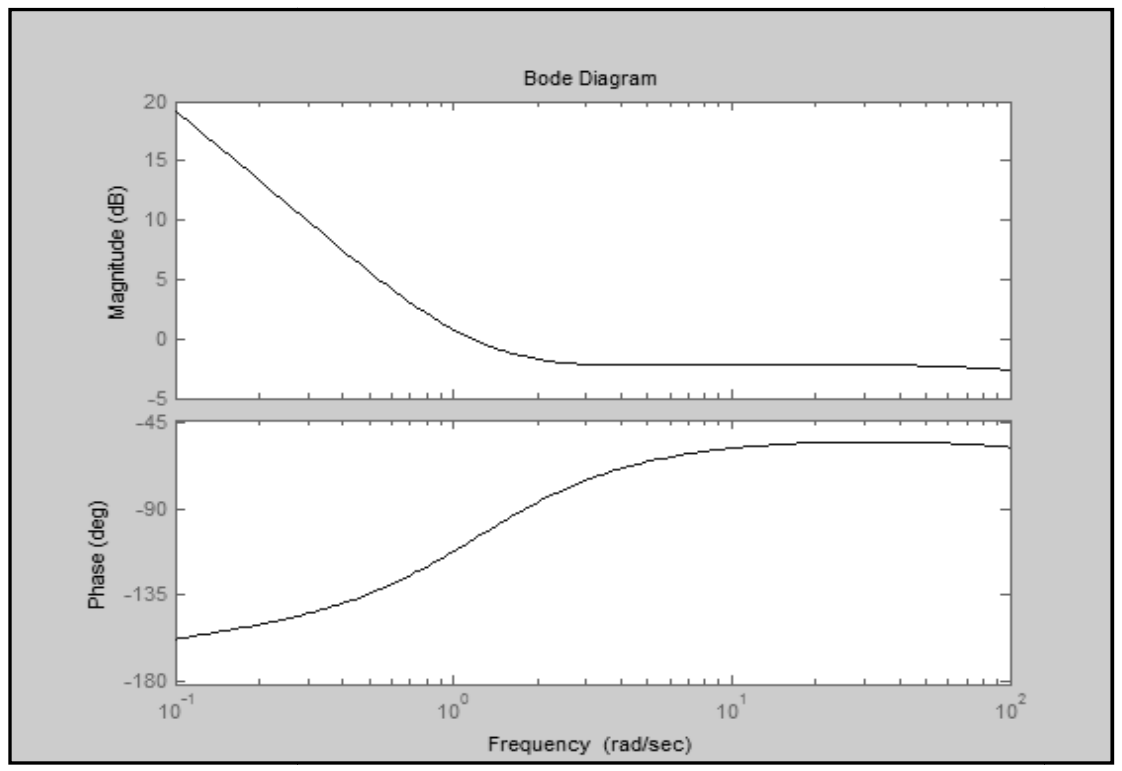

(b)

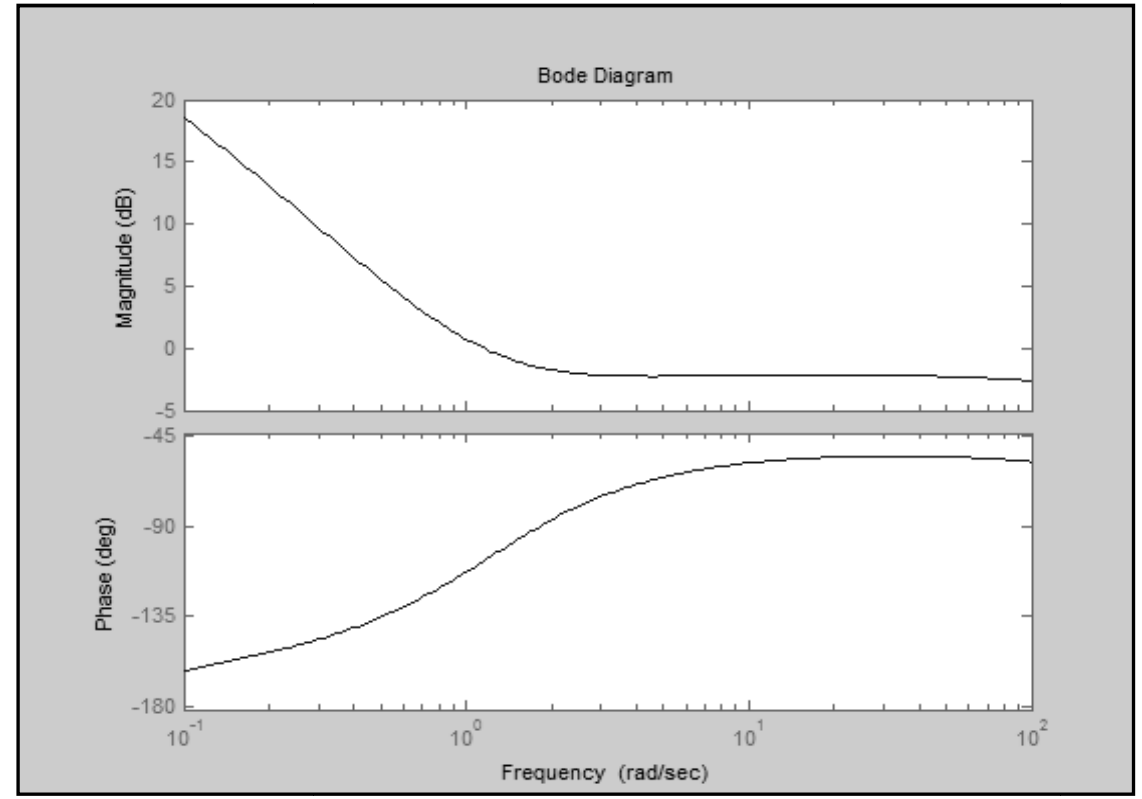

(c)

Figure 9 Continued. Frequency response obtained from FSF model of Example 2 for (a) Input/Output 1 (b) Input/Output 2 (c) Input/Output 3 and (d) Input/Output 4. 


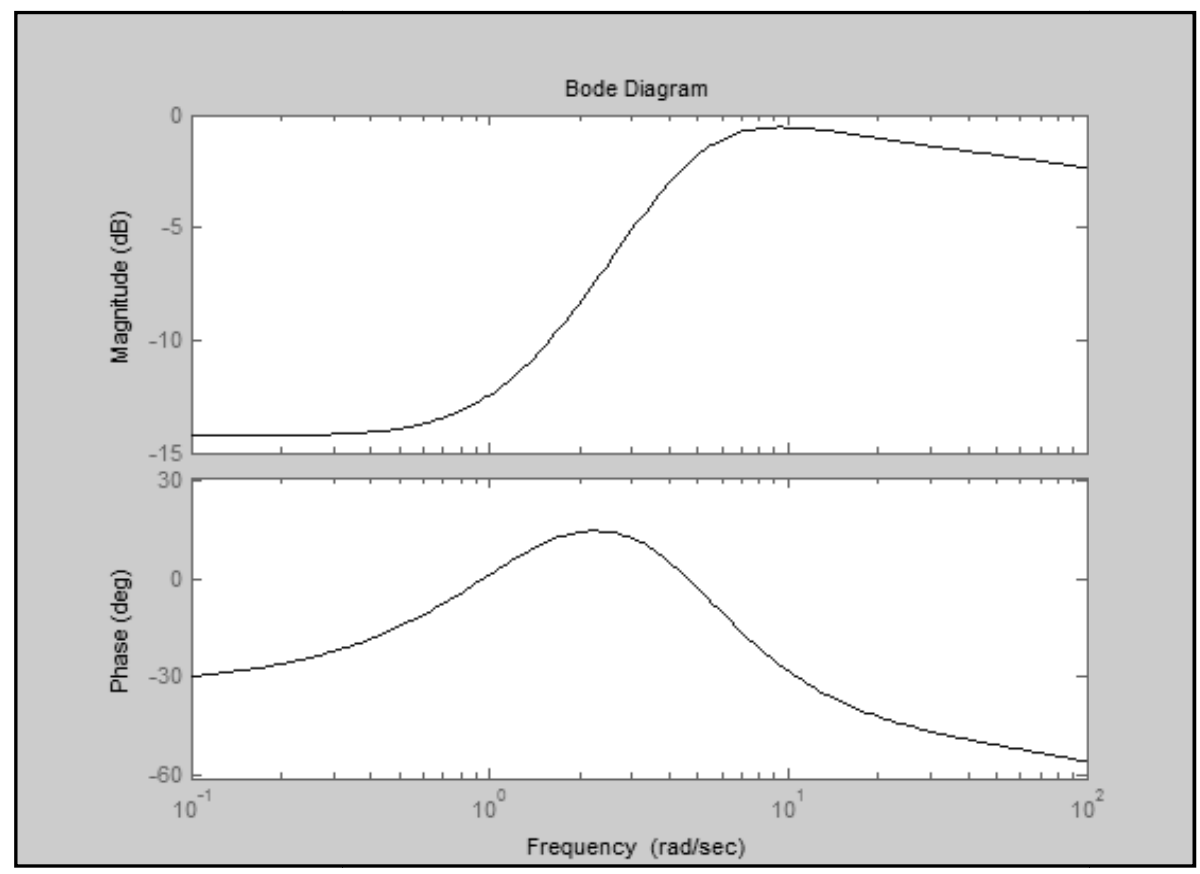

(d)

Figure 9 Continued. Frequency response obtained from FSF model of Example 2 for (a) Input/Output 1 (b) Input/Output 2 (c) Input/Output 3 and (d) Input/Output 4.

\subsection{Discussion}

As mentioned earlier, the MIMO FSF model involves a process of data compression in which the system step response in non-parametric form is obtained. The significance of this approximation lies in the fact that:

1. The large amounts of data that have been collected from the system can be reduced or compressed into smaller numbers of data.

2. The process frequency parameters that correspond to the higher frequency region of the system are neglected as the information content from that region normally has severe noise corruption.

3. A relative noise-free step response is obtained as compared to an actual step response test. Thus, it can be judged intuitively by the process engineer.

The number of compressed data must be chosen correctly so that no information about the system will be lost. To decide the amount of data that needs to be compressed, the step response estimate plot plays an important role. By looking at the step response estimation plot, we can see whether the number of 
compressed data is enough or too large or maybe too small. For instance, Figure 4 shows that for the $\mathrm{pH}$ neutralization system, 1000 data are already enough to be compressed. If, for example, we compressed these to 500 data only, the FSF model would be unable to capture the steady state mode of the system. Thus, part of the overall system information may be lost. Figure 10 shows the compression of Example 1 to 500 data.

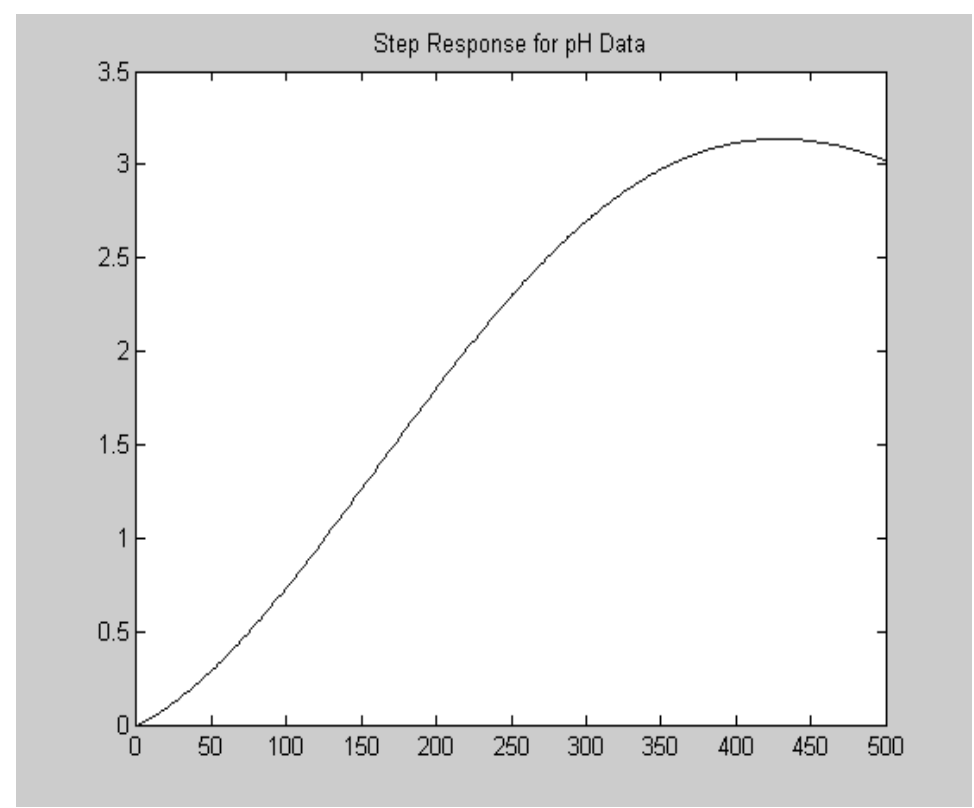

Figure 10 Step response obtained from FSF model of Example 1 with 500 compressed data.

On the other hand, if we compress the data from Example 1 to more than 1000 data, the data will still contain all the information about the system. However, a larger number of data will definitely affect computational time during the system identification process. Figure 11 shows the compression of the Example 1 data from 2000 data to 1500 data. In this figure, it can be seen that the step response estimate has reached the steady state condition at around 1000 data. Compression of data higher than this number should be ignored in order to optimize the computational time during the system identification procedure. Thus, in dealing with different types of plants and systems, one must make a wise judgment so as to make sure that all the information of the system is captured/modelled, and at the same time optimize the overall computational time. 


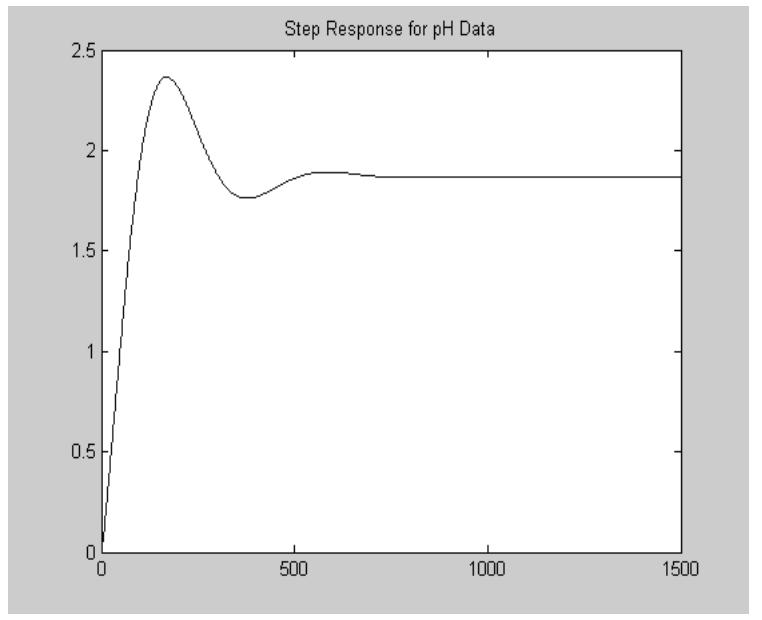

Figure 11 Step response obtained from FSF model of Example 1 with 1500 numbers of compressed data.

As mentioned in Section 2 and 3, the FSF approach assumes that the system is stable or asymptotically stable. Therefore, the produced step response will also become constant or asymptotically constant. Another assumption has to be made in order to use the FSF model for unstable systems. As discussed in [16], the same method can be used to unstable systems by introducing exponential weighting $\left(y_{a}(t)=e^{-a t} y(t) ; u_{a}(t)=e^{-a t} u(t) ; g_{a}(t)=e^{-a t} g(t)\right)$. And so the Laplace transformation will become $Y(s)=G(s) U(s) ; Y_{a}(s)=G_{a}(s) U_{a}(s)$ and $G_{a}(s)=G(s+a)$. If the unstable system, $G(s)$ (relating $u(t)$ to $y(t)$ ) has all poles $p_{i}$ such that $\operatorname{Re}\left(p_{i}\right)<a$, the system $G(s+a)$ is stable and relates the exponentially windowed signals (relating $u_{a}(t)$ to $y_{a}(t)$ ).

By having this, the performance index as in Eq. 15 has to be replaced with

$$
J_{b}(N, \hat{\Theta})=\sum_{k=0}^{N}\left|e^{b k \Delta}(Y-\Pi \Phi)\right|^{2}
$$

Using $b=a$, the effect of the exponential weighting will be taken care of, and all the data can be used. However, this method should be used with caution to avoid numerical rounding problems, and the minimum appropriate value of $a$ should be considered [16].

Another issue that can be raised is how to validate whether the step response estimates or the frequency response estimates that have been produced are accurate and informative enough to represent the true system. One solution is to have simulated data with a known model structure. Once the data are run using 
the FSF approach, the validation process can be performed by obtaining a mathematical model of the system using any standard system identification method. Standard verification tests (e.g. the mean square error (MSE) test, the $R^{2}$ test, best fit test, variance test etc.) in the system identification procedure can also be used as a way to verify its accuracy. Other than that, the developed model from the estimates can also be used together with the controller. The control performance can be observed, in which good control performance resembles a good model of the controlled system.

\section{Conclusion and Future Works}

Most real system applications involve the usage of a multiple number of input and output variables. This includes the large number of data to be collected and used for identification purposes. The novel approach of the MIMO FSF model proposed in this paper has three distinct advantages:

1. It investigates a true system application incorporating all possible input/output variables together.

2. It produces a clean and unbiased empirical model that can be used for system identification.

3. It reduces the amount of data for further study and analysis of the overall system.

For future works, the step and frequency response estimates (empirical model) can be used in identifying the mathematical model of both of these applications. The obtained model of the applications can be used for further research on control analysis. By having the model and observing the control performance, one may verify the efficacy of having the FSF filter prior to the system identification procedure as compared to direct use of the raw data to obtain the model of the system.

In addition, future work is also considered in setting up automated system configuration and data compression, in which assessing the optimal number of data to be compressed, and the tuning and the verification can be done simultaneously.

\section{Acknowledgments}

The authors' would like to acknowledge Universiti Sains Malaysia for the awarded short-term grant to support this project. Appreciation also goes to DaISy Identification Database for the data used in this project. 


\section{References}

[1] Mohd-Mokhtar, R., Aziz, M.H.R.A., Arshad, M.R. \& Hussain, N.A.A., Data Compression for Underwater Glider System Using Frequency Sampling Filters, Indian Journal of Geo-Marine Sciences, 40(2), pp. 227235, 2011.

[2] Mohd-Mokhtar, R., Aziz, M.H.R.A., Arshad, M.R. \& Hussain, N.A.A., Data Compression for Underwater Glider System Using Frequency Sampling Filters, in $3^{\text {rd }}$ International Conference on Underwater System Technology: Theory and Applications 2010 (USYS'10), Cyberjaya, Malaysia, pp. 18-23, 2010.

[3] Aziz, M.H.R.A. \& Mohd-Mokhtar, R., Multi Input Multi Output Frequency Sampling Filters for Real System Applications, Int. Conf. on Electrical Engineering and Informatics (ICEEI 2011), Bandung, Indonesia, 2011.

[4] Aziz, M.H.R.A. \& Mohd-Mokhtar, R., Identification of MIMO Magnetic Bearing System Using Continuous Subspace Method with Frequency Sampling Filters Approach, 37th Annual Conf. of the IEEE Industrial Electronics Society (IECON 2011), Melbourne, Australia, pp. 546-551, 2011.

[5] Mohd-Mokhtar, R., Aziz, M.H.R.A., Arshad, M.R. \& Hussain, N.A.A., Model Identification for Underwater Glider System Using Two Stage Identification Approach, ASEAN Symposium on Automatic Control (ASAC2011), Ho Chi Minh, Vietnam, pp. 92-97, 2011.

[6] Mohd-Mokhtar, R., Continuous Time State-space Model Identification with Application to Magnetic Bearing Systems, PhD thesis, School of Electrical and Computer Engineering, RMIT, Melbourne, Australia, 2008.

[7] Mohd-Mokhtar, R. \& Wang, L., 2-Stage Identification Based on Frequency Sampling Filters and Subspace Frequency Response, Elektrika: Journal of Electrical Eng., 11(2), pp. 27-33, 2009.

[8] Mohd-Mokhtar, R. \& Wang, L., 2-Stage Approach for Continuous Time Identification Using Step Response Estimates, in Proc. Of IEEE Int. Conf. on Systems, Man \& Cybernetics (SMC2008), Singapore, pp. 3183-3188, 2008.

[9] Bitmead, R.R. \& Anderson, B.D.O., Adaptive Frequency Sampling Filters, IEEE Trans. Circuits Syst., 28, pp. 524-533, 1981.

[10] Parker, P.J. \& Bitmead, R.R., Adaptive Frequency Response Identification, Proc. 26th IEEE Conf on Decision and Control, pp. 348-353, 1987.

[11] Stubberud, P.A. \& Leondes, C.T., A Frequency Sampling Filter Design Method which Accounts for Finite Word Length Effects, IEEE Trans. Sig. Process. pp. 189-193, 1984.

[12] Goberdhansingh, E., Wang, L. \& Cluett, W.R., Robust Frequency Domain Identification, Chem. Eng. Sci. 47, 1989-1999, 1992. 
[13] Cluett, W.R., Wang, L. \& Zivkovic, A., Development of Quality Bounds for Time and Frequency Domain Models: Application to The Shell Distillation Column, J. Process Control, 7, pp. 75-80, 1997.

[14] Wang, L. \& Cluett, W.R., From Plant Data to Process Control: Ideas for Process Identification and PID Design, London: Francis \& Taylor, 2000.

[15] Arifin, N., Wang, L., Goberdhansingh, E. \& Cluett, W.R., Identification of The Shell Distillation Column Using The Frequency Sampling Filter Model, Journal of Process Control, 5(2), pp.71-76, 1995.

[16] Gawthrop, P.J. \& Wang, L., Data Compression for Estimation of Physical Parameters of Stable and Unstable Systems, Automatica, 41, pp. 1313-1321, 2005.

[17] Wang, L., Gawthrop, P.J. \& Chessari, C., Indirect Approach to Continuous Time System Identification of Food Extruder, Journal of Process Control, 14, pp. 603-615, 2004.

[18] Wang, L. \& Cluett, W.R., Frequency-sampling Filters: An Improved Model Structure for Step-response Identification, Automatica, 33(5), pp. 939-944, 1997.

[19] Wang, L. \& Cluett, W.R., Use of PRESS Residuals in Dynamic System Identification, Automatica, 32(5), pp. 781-784, 1996.

[20] Moor, B.L.R.D. DaISy: Database for the Identification of Systems [Online]. Available: http://homes.esat.kuleuven.be/ smc/daisy/,2011 (Information accessed on $11^{\text {th }}$ Feb 2011).

[21] Pellegrinetti, G. \& Bentsman, J., Nonlinear Control Oriented Boiler Modeling - A Benchmark Problem for Controller Design, IEEE Trans. On Control Systems Technology, 4(1), pp. 57-64, 1996. 